\title{
Plant-associate interactions and diversification across trophic levels
}

\author{
Jeremy B. Yoder ${ }^{1,2, 凶}$, Albert Dang $^{1}$, Caitlin MacGregor ${ }^{1}$, and Mikhail Plaza ${ }^{1}$ \\ ${ }^{1}$ Department of Biology, California State University Northridge; Northridge, CA, 91330 \\ ${ }^{2}$ ORCID 0000-0002-5630-0921
}

Interactions between species are widely understood to have promoted the diversification of life on earth, but how interactions spur the the formation of new species remains unclear. Interacting species often become locally adapted to each other, but they may also be subject to shared dispersal limitations and environmental conditions. Moreover, theory predicts that different kinds of interactions have different effects on diversification. To better understand how species interactions promote diversification, we compiled published genetic data for host plants and intimately associated herbivores, parasites, and mutualists. We first tested whether host and associate population structures were correlated - an indication of associates locally adapting to hosts - and tested for confounding correlations with geographic distance or climate variation. We used Bayesian multiple regression to estimate the effect of host plant genetic differentiation on associate genetic differentiation over and above the confounding effects of geography and climate. We found that plant and associate genetic structures are indeed often congruent, but isolation by distance and by climate are also common. Multiple regressions established that the effect of host plants on associates is robust to accounting for geographic distance and climate. Finally, associate genetic structure was significantly explained by plant genetic structure more often in antagonistic interactions than in mutualistic ones. This supports a key prediction of coevolutionary theory, that antagonistic interactions promote diversity through local adaptation of antagonists to hosts, while mutualistic interactions promote diversity via the effect of hosts' geographic distribution on mutualists' dispersal.

Keywords: local adaptation, population structure, mutualism, herbivory, parasitism

$\mathbf{I}^{\mathrm{n}}$ nteractions between species have long been recognized as key drivers in the diversification of life on Earth (Agrawal and Zhang, 2021; Dar-

Correspondence: jeremy.yoder@csun.edu win, 1859; Ehrlich and Raven, 1964; Farrell et al., 1992; Grant, 1949; Hembry et al., 2014; Thompson, 2005). Intimate interactions in particular - those in which a parasite or mutualist spends much of its life in association with a single host individual - are implicated in elevated rates of diversification (Cruaud et al., 2012; Farrell, 1998; Futuyma and Agrawal, 2009; McKenna et al., 2019; Mitter et al., 1988), patterns of phylogenetic congruence between host and associate lineages (Althoff et al., 2012; Cruaud et al., 2012; Escudero, 2015; Liu et al., 2013), and host-associated differentiation within species (Althoff, 2008; Drès and Mallet, 2002; Peterson and Denno, 1998; Schneider et al., 2016; Stireman et al., 2006). However, it remains unclear how often selection created by interacting species directly contributes to the formation of reproductive isolation, and there is a building consensus that different forms of interaction have different effects on diversification.

There are multiple processes by which species interactions may promote diversification, operating at time scales ranging from a few growing seasons to millions of years (Agrawal and Zhang, 2021; De Vienne et al., 2013; Janz, 2011; Thompson, 2005). The classic escape-and-radiate model predicts cycles of alternating diversification - first in the hosts or victims, then in the associates or enemies - driven by the evolution of defenses and counter-defenses (Ehrlich and Raven, 1964; Futuyma and Agrawal, 2009; Janz, 2011). Escape-andradiate processes should result in associated clades within larger interacting lineages, such as Ehrlich and Raven's butterflies and their larval host plants 1964, but not necessarily congruence at lower levels of biological organization. This is because the diversification occurs asynchronously - a victim clade diversifies after "escaping" the association with the help of a new defense, then the antago- 
nist clade diversifies after overcoming that defense (Thompson, 2005).

At a smaller scale, intimately interacting species have also been predicted to show patterns of contemporaneous speciation (Forbes et al., 2009). Adaptation to an interacting species has been widely shown to create local adaptation and the beginnings of ecological speciation (Alstad 1998; Capelle and Neema 2005; Hanks and Denno 1994; Laine 2005; reviewed by Hargreaves et al. 2020; Hoeksema and Forde 2008; Runquist et al. 2020). However, evidence for speciation directly attributable to particular interactions has been surprisingly sparse (Althoff et al., 2014; Hembry et al., 2014). Within species, local adaptation driven by a species interaction should be detectable as ecological isolation, in which the population structure of one interacting species parallels that of the other (Nosil et al. 2003; Peterson and Denno 1998; Wang and Bradburd 2014; Fig. 1). Such correlated population structure is not proof positive of adaptation to an interacting species, though - it may also evolve if both species have similar dispersal limitations (Wright, 1943) or become locally adapted to variation in their shared environments (Futuyma and Peterson, 1985; Nuismer et al., 2010).

Furthermore, the nature of a species interaction probably determines whether or not it creates ecological isolation (Yoder, 2016). Antagonistic interactions such as predation or parasitism can create local arms races and cycles of allele frequencies or interacting phenotypes (Gomulkiewicz et al., 2000; Nuismer, 2006; Nuismer et al., 2010; Ridenhour and Nuismer, 2007; Yoder and Nuismer, 2010). These processes are expected to lead to local adaptation between populations of interacting species, and potentially co-diversification. In contrast, mutualistic interactions may often generate species-wide stabilizing selection, if the mutualists benefit from matching whatever traits mediate the interaction (Kiester et al., 1984; Yoder and Nuismer, 2010). In this case, correlated population structures would only arise via isolation by distance or local adaptation to shared environments (Nuismer et al., 2010), and theory in multiple frameworks has found that mutually beneficial interactions tend to promote less divergence or diversification than antagonistic interactions, under otherwise comparable circumstances (Kopp and Gavrilets, 2006; Maliet et al., 2020; Yoder and Nuismer, 2010).
Interactions between plants and the parasites, symbionts, and herbivores they host provide a potentially useful venue to examine congruence in the population structure of intimately interacting species, and to test contrasting predictions about the outcomes of different interaction types. Because they are a fundamental resource, plants are a substrate to which associated species must adapt, and as a limiting factor that may shape associates' biogeography (Futuyma and Peterson, 1985). Plants' impact on their associates' local adaptation and population genetic structure has been a subject of study for decades (Blakley, 1982; Drès and Mallet, 2002; Futuyma and Peterson, 1985; Matsubayashi et al., 2010; Peterson and Denno, 1998). However, most population genetic studies in this literature examine only the plant, or the associate - or focus on adaptation of associates to contrasting host species rather than adaptation of associate populations to host populations (or vice versa) (Drès and Mallet, 2002; Futuyma and Peterson, 1985; Matsubayashi et al., 2010; Rausher, 1983). These trends reflect the need to simplify study design and interpretation, but they mean that we may miss intraspecific selection dynamics that are the ultimate cause of larger-scale evolutionary patterns (Thompson, 2005, 2013). Fortunately, studies of within-species population genetic data for plants and their associates are becoming both more practicable and more common. Compilation and synthesis of such studies can reveal patterns about the evolutionary importance of associates' local adaptation to host plants, and may also guide future work in this line of inquiry by revealing biases and gaps in the range of study systems examined so far.

Here, we examine the congruence of population genetic structure in plants and associated species across a dataset compiled from published studies. We test the hypothesis that host plants' population structure predicts the population structure of associate species using meta-analysis of correlations between genetic, geographic and environmental distances, then use Bayesian multiple linear regression to examine the effect of host plant population structure on their associates' genetics, over and above confounding effects of geography and climate. We find evidence that plant population structure can strongly contribute to associate population structure, both in general and in antagonistic interactions specifically. However, in mutu- 
A

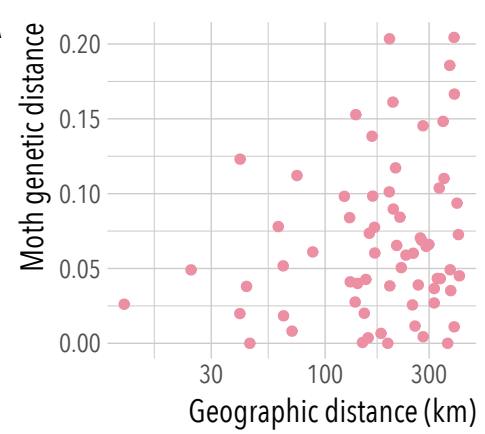

C

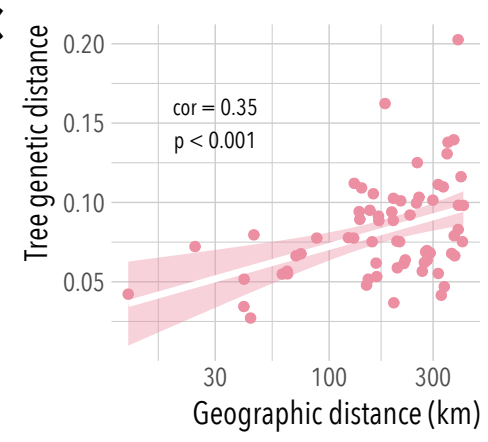

B

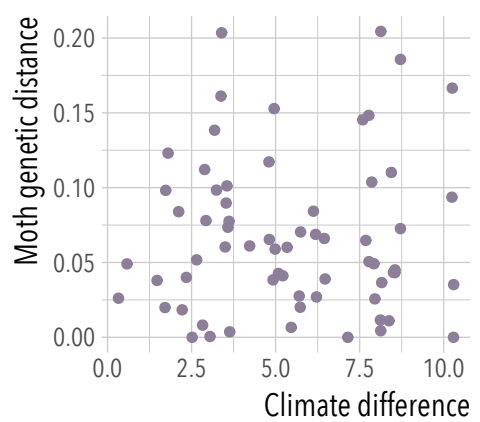

D

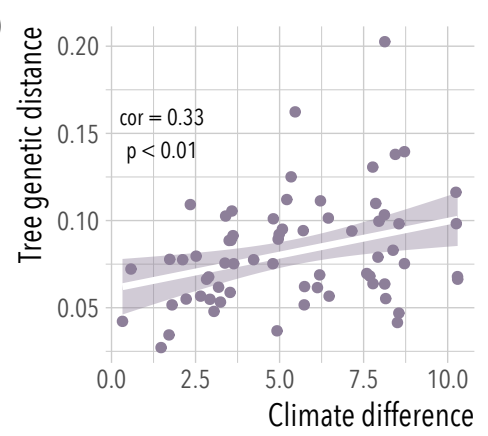

E

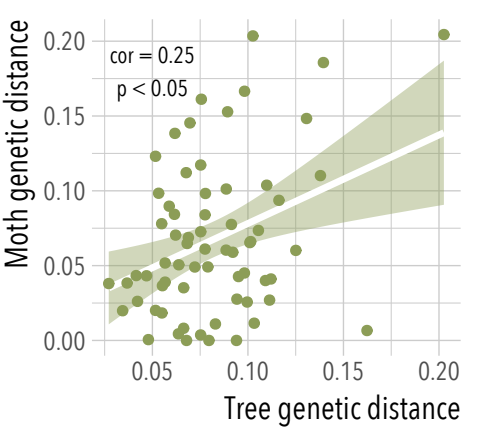

Fig. 1. An example with data from western Joshua tree (Yucca brevifolia) and the pollinating, seed-feeding yucca moth Tegeticula synthetica (Yoder et al., 2013). Moth genetic distances $\left(G_{S T} /\left(1-G_{S T}\right)\right.$ ) are not significantly correlated with geographic distance (A) or with climate differences between sites (B); but Joshua tree genetic distances show significant correlations with distance (C; Spearman's $\rho=0.41$, bootstrap $p<0.001$ ) and with climate (D; Spearman's $\rho=0.33, p<0.01)$. A significant positive correlation between Joshua tree genetic distance and moth genetic distance $(\mathrm{E} ; \rho=0.35, p<0.05)$ may be consistent with local adaptation to the trees creating ecological isolation in the moths.

alistic interactions, we find that associates' population structure is more often shaped by shared geographic isolation than by local adaptation to individual plant populations. We discuss these results in light of coevolutionary theory, and suggest future directions for population genetic studies of plant-associate interactions as robust genetic data becomes more accessible for non-model organisms.

\section{Methods}

Literature search. We compiled papers reporting population genetic data for plants and associated species from our personal collections, and using the Google Scholar search engine (scholar.google.com). We searched varying combinations of keywords referring to population structure (structure, Fst, or "pairwise Fst"), keywords referring to species interactions (mutualism, pathogen, parasite, symbiont, pollinator, herbivore, or coevolution), and always included the keyword plant. All keyword sets returned at least 1,500 results, and many had more than 20,000; we reviewed titles and abstracts for the first 200 papers returned in each search, and generally found that relevant results were not found after the first 100 . We found, in total, 23 papers discussing or reporting population genetic data for both a plant species and at least one associated mutualist, herbivore, or pathogen. Of these, we retained 14 papers that provided either pairwise genetic differentiation estimates, or genetic data that would support such estimates, for both species at four or more geographically distinct sites (Table 1). As a final check on the thoroughness of our literature search, we used Google Scholar to examine all papers citing the final set of studies, and this search did not return any additional studies for inclusion.

Data compilation. We assembled our working dataset and conducted all analyses in $\mathrm{R}$ (version 4.0.0, R Core Team 2020). For analysis we required pairwise genetic, climatic, and geographic 
distances for both a plant and one or more associated species, across the same samplings sites. Several of the papers in our final set reported these values directly, either in the main text or as supplementary information. For papers that did not report these values, we drew on supporting data to estimate pairwise necessary distances. The type of genetic marker used varied by study and even between plants and associates in the same study (Table 1), reflecting the challenge, until recently, of obtaining similar-quality genetic data for two interacting non-model species. The most common marker type was simple sequence repeat markers (SSRs, or microsatellites), with amplified fragment length polymorphisms (AFLPs) and allozyme polymorphisms represented in multiple cases as well. Where the supporting data for papers provided genetic data but not pairwise genetic distances, we used GenoDive (version 3.0, Meirmans 2020; Meirmans et al. 2004) to estimate genetic distances, either as $F_{S T}$ for SNP data, $G_{S T}$ for SSR or other fragment polymorphism data, or Jost's D (Jost 2008) for one case of haplotype sequence data from bacteria (Harrison et al., 2017).

Where papers provided sampling location data, either as latitude and longitude or as maps with sufficient detail to determine latitude and longitude, we used the locations to calculate greatcircle distances between sampling sites, in kilometers, with the rdist.earth() function provided in the fields package (Nychka et al. 2017). We extracted Bioclim climate data (Woldclim version 2.1, averages from 1970-2000; Fick and Hijmans 2017; Hijmans 2020) for sampling locations, then calculated climate differences between sampling sites by performing principle components analysis on site climate values and calculating between-site Euclidean distances in PCA space, using base $\mathrm{R}$ functions. Four studies representing five plant-associate pairs (Anderson et al. 2004; Jerome and Ford 2002; Magalhaes et al. 2011; Ren et al. 2007) provided geographic distances between sampling sites but insufficient supporting information to infer sampling site locations; in these cases we used reported geographic distances and performed analyses without climate as a variable.

Analyses. To describe relationships among genetic, environmental (climatic), and geographic distances, we first calculated simple correlations between all possible pairwise combinations of distance metrics for each plant-associate pair, as
Spearman rank correlations. We used the base10 logarithm of geographic distances, and the standard transformation of genetic distances as $D_{\text {genetic }} /\left(1-D_{\text {genetic }}\right)$ (Rousset, 1997). To estimate confidence intervals around pairwise correlations, we bootstrapped the distance data, using the bootstraps () function of the rsample package (Kuhn et al., 2020), with 1000 replicates.

To test whether host plant population structure creates ecological isolation for associates while accounting for shared geography and environmental factors, we fitted multiple linear regressions predicting associate genetic distances with additive linear effects of geographic distance, climate distance (if available), and host plant genetic distance. To cope with the highly non-normal distribution of the data, we fitted models using the Bayesian framework implemented in the brms package (Bürkner, 2018, 2017), with the response modeled as a zero-inflated beta distribution.

Finally, to summarize the overall strength of the pairwise distance correlations, or estimated predictor effects in the multiple linear regressions, we performed meta-analysis using the random-effects methods for correlations (the metacor() function) and mean effect estimates (the metamean () function) provided in the meta package (Balduzzi et al., 2019). To test for differences between antagonistic and mutualistic interactions, we included interaction types as a grouping variable in each random-effects meta-analysis. Associate taxa proved to be widely and unevenly phylogenetically dispersed, including a bacterium, a plant, and multiple insects, but also with three cases in which congeners or the same species were represented in multiple pairs (Table 1: Arceuthobium, Macropis, and Tegeticula). Because of the highly skewed range of phylogenetic distances among associates, we did not employ a formal control for phylogenetic non-independence in our meta-analyses, but we tested the robustness of our conclusions by systematically re-running each meta-analysis with each possible permutation of the dataset created by excluding one member from each of these three pairs of close relatives.

\section{Results}

Our literature search obtained 14 papers reporting population genetic data from 18 plant-associate pairs (Table 1). Six of the 18 pairs had antagonistic interactions, with the associates being herbivores 
Table 1. Plant-associate interactions in our compiled dataset, the broad type of each associate, the number of sampling sites reported, and the type of genetic markers used for each plant and associate

\begin{tabular}{|c|c|c|c|c|c|}
\hline \multirow[b]{2}{*}{ Plant/ Associate } & \multicolumn{2}{|l|}{ Associate } & \multicolumn{2}{|l|}{ Markers for } & \multirow[b]{2}{*}{ Source } \\
\hline & type & Sites & Plant & Associate & \\
\hline Camellia japonica/ Curculio camelliae & antagonist & 6 & SSR & sequence & Toju et al. (2011) \\
\hline Ficus hirta/ Valisia sp. & mutualist & 30 & SSR & SSR & Yu et al. (2019) \\
\hline Ficus pumila/ Wiebesia sp. & mutualist & 24 & SSR & SSR & Liu et al. (2013) \\
\hline Lysimachia vulgaris/ Macropis europaea & mutualist & 45 & AFLP & AFLP & Triponez et al. (2015) \\
\hline L. vulgaris/ M. fulvipes & mutualist & 17 & AFLP & AFLP & Triponez et al. (2015) \\
\hline Medicago lupulina/ Ensifer sp. & mutualist & 28 & SNP & sequence & Harrison et al. (2017) \\
\hline Pinus banksiana/ Arceuthobium americanum & antagonist & 11 & AFLP & AFLP & Jerome and Ford (2002) \\
\hline Roridula gorgonius / Pameridea roridulae & mutualist & 14 & allozyme & allozyme & Anderson et al. (2004) \\
\hline Silene latifolia/ Hadena bicruris & mutualist & 9 & SSR & SSR & Magalhaes et al. (2011) \\
\hline Vachellia drepanolobium/ Crematogaster nigriceps & mutualist & 9 & SNP & SNP & Boyle et al. (2019) \\
\hline V. drepanolobium/ Tetraponera penzigi & mutualist & 11 & SNP & SNP & Boyle et al. (2019) \\
\hline Yucca brevifolia/ Tegeticula synthetica & mutualist & 13 & SSR & SSR & Yoder et al. (2013) \\
\hline Y. jaegeriana/ T. antithetica & mutualist & 10 & SSR & SSR & Yoder et al. (2013) \\
\hline
\end{tabular}

or parasites, and the others were mutualistic interactions such as pollination, protection, or nutrient exchange. In 15 pairs, the associate was an insect; two pairs consisted of the parasitic plant Arceuthobium americanum on different conifer host species, and the final pair was a nitrogen-fixing rhizobial bacterium, genus Ensifer, and its legume host Medicago lupulina. In addition to the two data sets with A. americanum as the associate, cuckoo bees (genus Macropis) and yucca moths (genus Tegeticula) were each represented in two host-associate pairs.

Correlations with genetic distances. Pairwise correlations between genetic and geographic distance (i.e., isolation by distance), between genetic and climate distance (isolation by climate), and between genetic distances for plants and associate species (isolation by host) varied considerably across plant-associate pairs (Fig. 2). Host plants showed significant isolation by distance (Spearman rank correlation, $\rho>0$ and bootstrap $p<0.05$ ) in 10 of 18 pairs, and significant isolation by climate differences in 9 of 13 pairs for which climate data was available. Associates showed significant isolation by geographic distance in 7 of 18 pairs, and isolation by climate in 5 of 13 pairs.

Plant and associate genetic distance were significantly correlated, potentially consistent with associates experiencing isolation by host plant, in 10 of 18 plant-associate pairs - in 4 of the 6 antagonistic interactions, and 6 of the 12 mutualistic interactions. For one pair, the loosestrife Lysimachia vulgaris and the pollinating bee Macropis europaea, we found a significant negative correlation between genetic distances; this pattern is difficult to interpret biologically, but it is also reported in the original study by Triponez et al. (2015). Across the 18 plant-associate pairs, significant correlations between plant and associate genetic structure were not consistently seen in conjunction with isolation by distance or isolation by climate in either plants or associates (bootstrap $p>0.05$ in all cases).

The general signal revealed by meta-analysis was also that plant and associate genetic distances are significantly and positively correlated (Fig. 2; pooled estimate of Spearman's $\rho=0.26,95 \%$ confidence interval from 0.13 to 0.37 ). The meta-analytic pooled correlation between plant and associate genetic distances was also significantly greater than zero within antagonistic and mutualistic interactions (for antagonistic interactions, pooled $\rho=0.36$, $95 \%$ CI 0.22 to 0.48 ; for mutualistic, $\rho=0.21,95 \%$ CI 0.06 to 0.34 ). Although the mean correlation was somewhat greater for antagonistic interactions than for mutualistic interactions, this difference was not greater than expected by chance $(p=0.13$ 


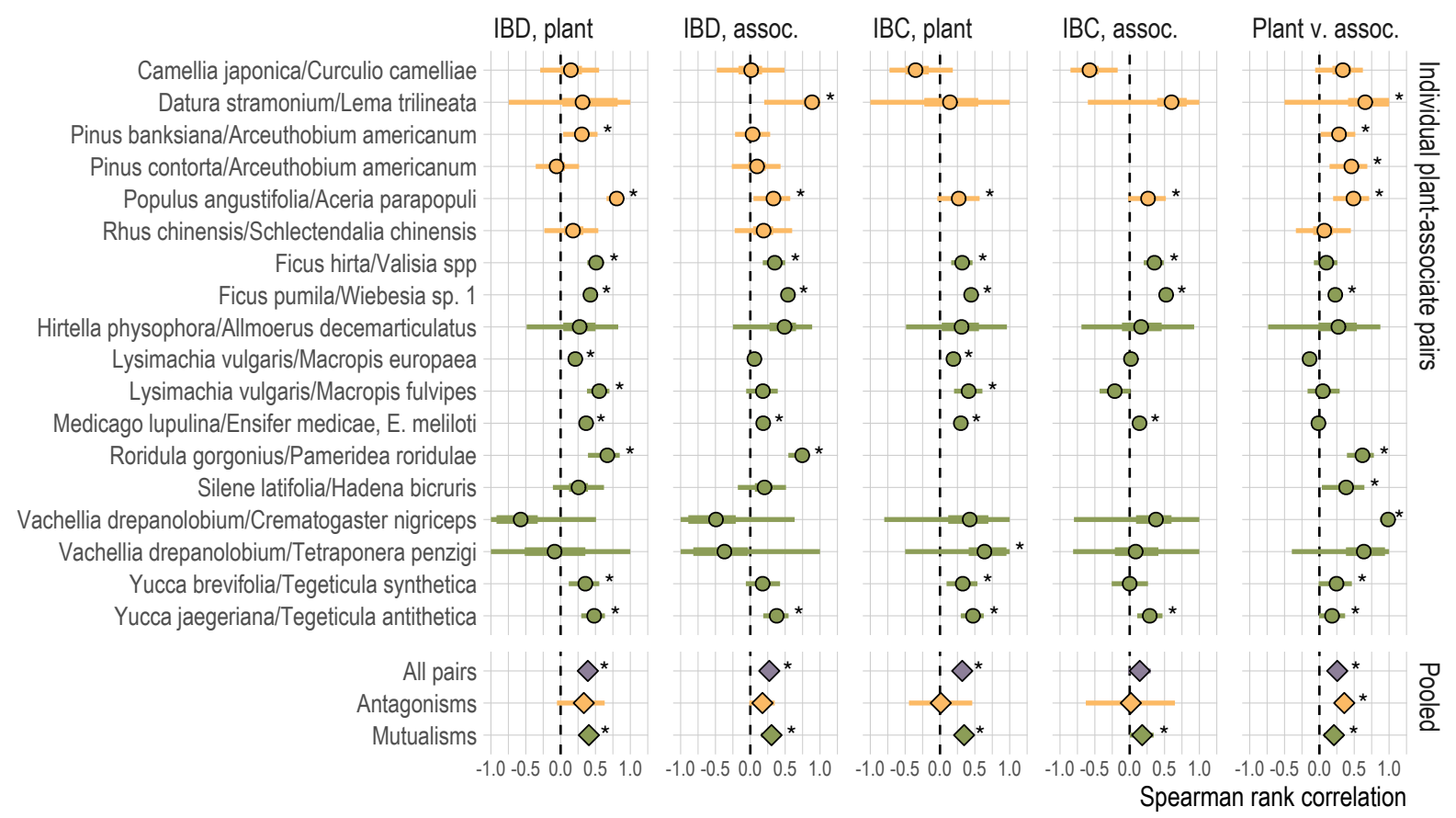

Fig. 2. Correlations between genetic, geographic, and climate distances in the plant-associate datasets we compiled, for each of 17 individual plant-associate pairs and for meta-analytic summaries across mutualisms, antagonisms, and all pairs. For individual pairs, points indicate Spearman rank-sum correlation $\rho$, bars give $95 \%$ (thin) and $50 \%$ confidence (thick) intervals based on 1000 bootstrap replicates. For meta-analytic pooled estimates, points give estimated mean and bars give $95 \%$ confidence intervals. Asterisks indicate correlations greater than zero with bootstrap $p<0.05$. Antagonistic interactions are colored yellow, mutualistic ones green.

for group differences in the random-effects metaanalysis). Excluding closely related associates from the dataset did not qualitatively alter any of these results. Overall, this is consistent with associates experiencing widespread isolation by host plant, across different types of interactions.

However, meta-analysis also found correlations with distance and climate that may be confounded with the correlations in genetic distances see above. Meta-analysis across all plant-associate pairs found significant isolation by distance for plants and associates (pooled $\rho=0.40,95 \%$ CI 0.30 to 0.49 for plants; $\rho=0.28,95 \%$ CI 0.16 to 0.40 for associates) and significant isolation by climate for plants ( $\rho=0.34,95 \%$ CI 0.26 to 0.41 for plants) but not associates ( $\rho=0.14,95 \%$ CI -0.02 to 0.30 for associates). These results were generally robust to pruning closely related associates, but removing data from Macropis europaea and Tegeticula antithetica had the effect of increasing the mean correlation between associate genetic distance and climate enough to be consistent with significant isolation by climate.
Meta-analysis of antagonistic interactions did not find significant correlations consistent with isolation by distance in either plants or associates $(\rho$ $=0.33,95 \%$ CI -0.05 to 0.63 for plants; $\rho=0.17$, $95 \%$ CI -0.01 to 0.35 for associates), nor significant isolation by climate $(\rho=0.01,95 \%$ CI -0.45 to 0.46 for plants; $\rho=0.02,95 \%$ CI -0.63 to 0.65 for associates). However, meta-analysis of mutualistic interactions found significant isolation by distance $(\rho$ $=0.41,95 \%$ CI 0.31 to 0.49 for plants; $\rho=0.30,95 \%$ CI 0.15 to 0.44 for associates) and significant isolation by climate $(\rho=0.35,95 \%$ CI 0.26 to $0.43 ; \rho=$ $0.18,95 \%$ CI 0.00 to 0.34 ) in both plants and associates. These differences between interaction types were not greater than expected by chance in the random effects model, however $(p>0.05$ for both isolation by distance and isolation by climate). Excluding closely related associates did not change these qualitative results.

Multiple linear regressions. Bayesian multiple linear regressions explaining associate population structure by the joint effects of geographic dis- 


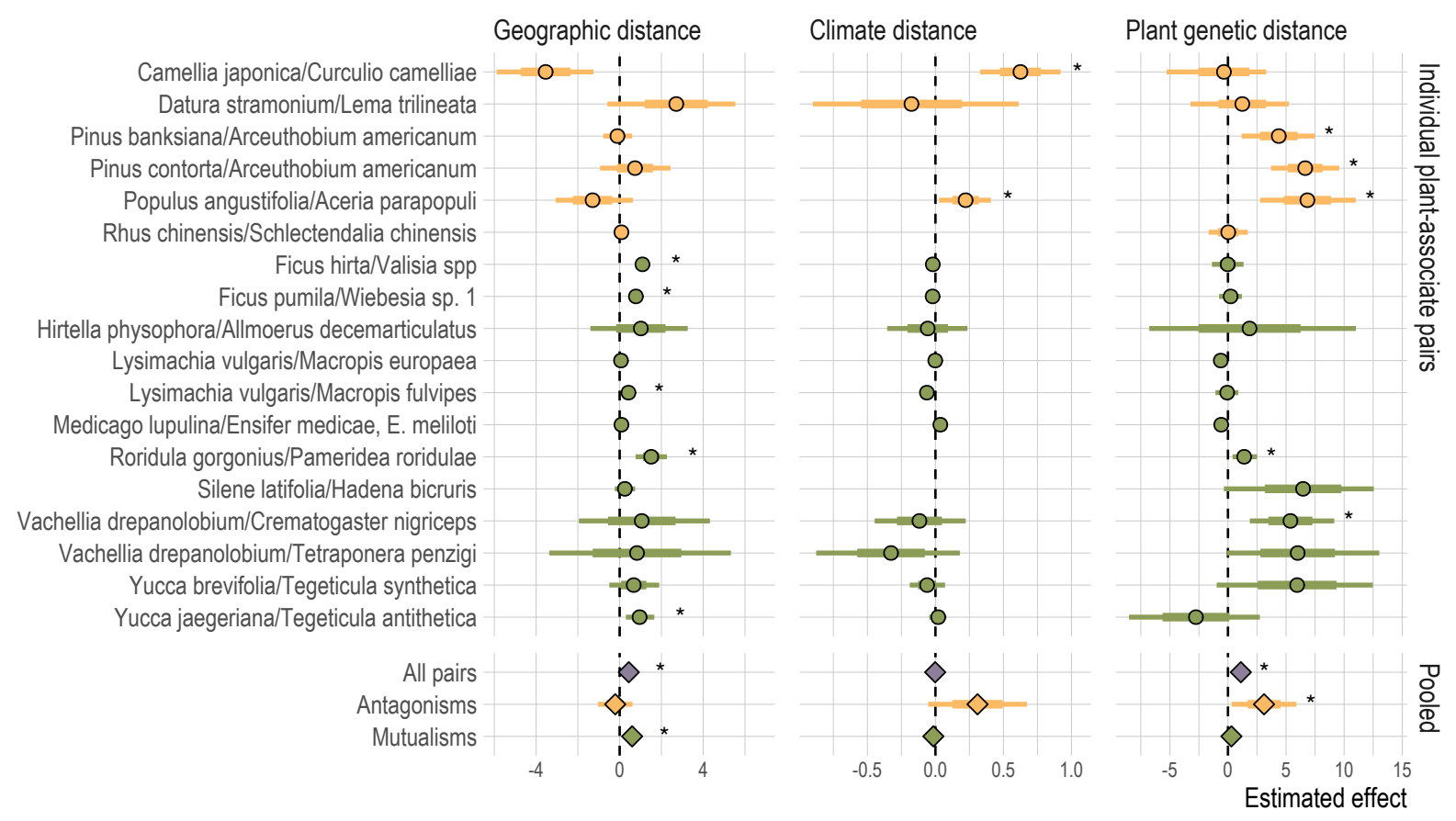

Fig. 3. Estimated effects of geographic distance, climate distance, and host plant genetic distance on associate genetic distance in Bayesian multiple linear regressions for each of 18 individual plant-associate pairs; and meta-analytic summaries of the same effect estimates across mutualisms, antagonisms, and all pairs. Points indicate posterior mean effect, bars give 95\% (thin) density intervals and standard error (thick). Asterisks and colors follow Fig. 2.

tance, climate (where available), and host plant genetic distance returned results that generally paralleled patterns in the simple pairwise correlations (Fig. 3). Correlations between plant and associate genetic distances strongly predicted multiple regression estimates of the effect of plant genetic distance on associate genetic distance (Spearman's $\rho$ $=0.69, \mathrm{p}=0.002$; Figs 2,3). However, multiple regression models included a significantly nonzero effect of isolation by host plant in only half of the plant-associate pairs that had showed a significant correlation between plant and associate genetic distances: the estimated effect of plant genetic distance on associate genetic distance was greater than zero, with a $95 \%$ posterior density interval that did not contain zero, in just 5 plantassociate pairs ( $28 \%$ of the 18 ; Fig. 3). In general, effect estimates for isolation by climate were smaller than effect estimates for isolation by geographic distance or isolation by host plant (Fig 3).

Meta-analysis across all plant-associate pairs found a significant effect of plant genetic distance (Fig. 3; estimate $=1.11,95 \%$ CI 0.33 to 1.89 ) and a significant effect of geographic distance (estimate
$=0.44,95 \%$ CI 0.17 to 0.71$)$; but the meta-analytic mean effect of climate was not significantly different from zero (estimate $=-0.001,95 \%$ CI -0.04 to 0.03 ). Excluding close relatives did not change these qualitative results. This is consistent with associates generally experiencing both isolation by distance and isolation by host plant, but not isolation by climate.

Meta-analysis across all antagonistic pairs found no significant isolation by distance (estimate $=-0.21,95 \%$ CI -1.04 to 0.62 ) but significant isolation by host plant (estimate $=3.11,95 \%$ CI 0.32 to 5.89); whereas meta-analysis across mutualist pairs found significant isolation by distance (estimate $=0.59,95 \%$ CI 0.29 to 0.89 ) but no significant isolation by host plant (estimate $=0.30$, $95 \%$ CI -0.38 to 0.97 ). The random-effects metaanalysis found that this difference in isolation by distance was only marginally significant $(p=0.07)$; but the difference in isolation by host plant was greater than expected by chance $(p=0.05)$. Excluding close relatives did not change the qualitative results, but did impact the random-effects assessment of differences between interaction types. 
With the Pinus banksiana/Arceuthobium americanum pair excluded, meta-analyses found that antagonistic interactions had significantly lower isolation by distance than mutualistic ones; while excluding any of the closely related associates resulted in meta-analysis finding that interaction types did not significantly differ in isolation by host plant ( $p>0.05$ in all cases). Overall, this is consistent with antagonistic associates experiencing isolation by host plant but not by geographic distance, while mutualistic associates experience isolation by distance but not by host plant.

\section{Discussion}

A wide range of experimental and observational evidence describes the ways in which plants may shape the evolutionary history of species that depend upon them. Population genetic data of plants and associates interacting across a shared landscape provides a look at local adaptation, the process by which the ecological dynamics of plantassociate interactions are translated into global patterns of biodiversity (Drès and Mallet, 2002; Futuyma and Peterson, 1985; Hembry et al., 2014; Peterson and Denno, 1998). Plants and their associates disperse and evolve across a shared landscape, and face the same geographically varying environments, even as associates adapt to their host plants (Futuyma and Peterson, 1985; Thompson, 2005); and different types of interactions may create differing plant-associate selective dynamics, and therefore different evolutionary outcomes (Maliet et al., 2020; Nuismer et al., 2010; Yoder and Nuismer, 2010).

Compiling published population genetic data for plant-associate pairs, we find evidence that host plants shape their intimate associates' evolution through the effects of shared geographic distribution and through associates' local adaptation to host plants - but generally not through adaptation to shared environmental conditions, specifically climate (Fig. 3). Simple pairwise correlations between genetic distances and geographic or climate distances are consistent with effects of isolation by distance and isolation by climate in both plants and associate species (Fig. 2, meta-analysis across all pairs). However, in a multiple regression framework accounting for the confounding among geography, climate, and host plant genetic distances, we see that climate differences have much smaller impacts on associates' genetic differentia- tion than either geographic distance or host plant genetic distances (Fig. 3, meta-analysis across all pairs). Accounting for geographic distance, climate, and plant genetic distance simultaneously in the multiple regression framework also substantially reduces the number of cases in which we see significant effects of plant population structure on the population structure of associate taxa.

Global patterns in plant-associate interactions. Overall, these results demonstrate that associates' population structures often reflect those of their host plants, and that this pattern arises both because of associates' local adaptation to hosts (ecological isolation by host population) and because of the effects of shared geography (isolation by distance). We find that the meta-analytic mean effect of climate differences on associate genetic distance is non-significant, reflecting the fact that we found significant effects of climate in only two of 13 plant-associate pairs for which we could obtain climate data at sampling sites (Fig. 3). On the one hand, it may be somewhat surprising that adaptation to shared environments does not contribute to congruence in plant-associate population structure; but on the other it may be that climate differences are sufficiently conflated with geographic distance and host plant population structure that including this additional variable may not be informative. Recent systematic reviews and metaanalysis have also found that local adaptation to abiotic factors is often weaker than local adaptation to biotic interactions (Hargreaves et al., 2020; Runquist et al., 2020), and the pattern we see here is consistent with these results.

Perhaps most excitingly, we find contrasting trends in different types of plant-associate interaction. Antagonistic associates, primarily herbivores and parasites, show a significant overall effect of isolation by host plant, but not significant isolation by geographic distance (Fig 3, metaanalysis across antagonisms); whereas mutualistic associates such as pollinators and nitrogen-fixing symbionts show significant isolation by distance, but not isolation by host plant (Fig 3, meta-analysis across mutualisms). This result aligns with the predictions of coevolutionary theory in multiple frameworks, which have found that antagonistic interactions mediated by phenotype differences or inverse matching can promote diversification of associates, whereas mutualistic interactions mediated by matching of host and associate traits tend 
to create stabilizing selection across populations (Kopp and Gavrilets, 2006; Maliet et al., 2020; Yoder and Nuismer, 2010). In the former case, the arms-race and inverse-frequency-dependent dynamics of antagonistic interactions should often mean that associates are adapted to their local host populations (Gomulkiewicz et al., 2000; Nuismer et al., 2007; Ridenhour and Nuismer, 2007) - and therefore show isolation by host plant, as we find. In the latter case - mutualisms - we would expect that associates' variation across populations would be driven more by drift and spatial isolation than by local adaptation to host plant populations, because hosts and associates would generally be selected to remain compatible across populations (Kiester et al., 1984; Yoder and Nuismer, 2010).

Opportunities for future work. The results of our literature search reveal considerable opportunities for future studies of population genetic patterns across trophic levels. Although population genetic tools have been applied to the broad subject of plant-associate diversification for decades, we find just 14 papers reporting data for both a plant and at least one intimate associate species 11 published in the last 10 years. The majority of these studies use codominant elecrophoretic markers, which have relatively poor resolution to characterize diversity and differentiation, compared to modern DNA sequencing capacities; and this is reflected in the wide range of precision we find when we estimate correlations between genetic, geographic, and environmental distances (Fig 2). We expect that future studies of this type will benefit greatly from the increased accessibility of genomewide sequence data (Andrews et al., 2016; Davey et al., 2011).

Finally, 15 of 17 unique associate taxa in our compiled dataset are insects. This pattern is not surprising in light of the historical focus on angiosperm-insect co-diversification (Ehrlich and Raven, 1964; Farrell, 1998; Farrell et al., 1992; Futuyma and Peterson, 1985; Peterson and Denno, 1998). However, many of the same reasons to expect that insect herbivores and mutualists should evolve in response to their host plants apply to symbiotic fungi (e.g. Escudero, 2015), bacteria (Harrison et al., 2017), and even parasitic or commensal plants (Jerome and Ford, 2002; Schneider et al., 2016) - other cases in which a single associate individual lives most of its life on one host, and in which associates' geographic dispersal is limited by hosts' distribution. Moreover, plants are far from alone in hosting parasitic and mutualistic associates in intimate interactions; it is easy to imagine a version of the present analysis examining animal hosts and microbial symbionts, parasitic nematodes, or pathogenic viruses. One of the clearest opportunities for further contributions to this line of inquiry is the expansion of its taxonomic scope.

Conclusions. Compiling population genetic data for plants and closely associated taxa, we find support for the widespread understanding that intimate interactions across trophic levels create diversifying natural selection. Although both antagonistic and mutualistic associates frequently evolve population structures that parallel those of their host plants, our analysis finds that antagonists are more likely to become locally adapted to individual host populations, while mutualists are more likely to be affected by the effects of sharing geographic distributions with their hosts. Future studies of plant-associate population genetics will, we hope, expand the taxonomic scope of this data, to further illuminate how plant-associate interactions have fueled the diversification of life on Earth.

\section{Acknowledgements}

We thank Christopher I. Smith and John N. Thompson for helpful comments on earlier drafts of this paper. Support was provided by start-up funds from California State University Northridge and the U.S. National Science Foundation (DEB, 2001180).

\section{Data availability}

Supporting data will be made publicly available upon final publication of this manuscript.

\section{References}

Agrawal, A. A., and X. Zhang. 2021. The evolution of coevolution in the study of species interactions. Evolution pages 1594-1606.

Alstad, D. 1998. Population Structure and the Conundrum of Local Adaptation. Pages 3-21 in Genetic Structure and Local Adaptation in Natural Insect Populations. Springer US, Boston, MA.

Althoff, D. M. 2008. A test of host-associated differentiation across the 'parasite continuum'in the tri-trophic interaction among yuccas, bogus yucca moths, and parasitoids. Molecular Ecology 17:3917-3927. 
Althoff, D. M., K. a. Segraves, and M. T. J. J. Johnson. 2014 Testing for coevolutionary diversification: Linking pattern with process. Trends in Ecology and Evolution 29:82-89.

Althoff, D. M., K. A. Segraves, C. I. Smith, J. Leebens-Mack, and O. Pellmyr. 2012. Geographic isolation trumps coevolution as a driver of yucca and yucca moth diversification. Molecular Phylogenetics and Evolution 62:898-906.

Anderson, B., I. Olivieri, M. Lourmas, and B. A. Stewart. 2004. Comparative population genetic structures and local adaptation of two mutualists. Evolution 58:1730-1747.

Andrews, K. R., J. M. Good, M. R. Miller, G. Luikart, and P. A. Hohenlohe. 2016. Harnessing the power of RADseq for ecological and evolutionary genomics. Nature Reviews Genetics 17:81-92.

Balduzzi, S., G. Rücker, and G. Schwarzer. 2019. How to perform a meta-analysis with R: a practical tutorial. EvidenceBased Mental Health pages 153-160.

Blakley, N. 1982. Biotic unpredictability and sexual reproduction: Do aphid genotype-host genotype interactions favor aphid sexuality? Oecologia 52:396-399.

Boyle, J. H., D. Martins, P. M. Musili, and N. E. Pierce. 2019. Population genomics and demographic sampling of the antplant Vachellia drepanolobium and its symbiotic ants from sites across its range in east Africa. Frontiers in Ecology and Evolution 7:1-49.

Bürkner, P.-C. 2018. Advanced Bayesian multilevel modeling with the R package brms. The R Journal 10:395.

Bürkner, P.-C. C. 2017. brms: An R package for Bayesian multilevel models using Stan. Journal of Statistical Software 80:128.

Capelle, J., and C. Neema. 2005. Local adaptation and population structure at a micro-geographical scale of a fungal parasite on its host plant. Journal of Evolutionary Biology 18:1445-1454.

Cruaud, A., N. Ronsted, B. Chantarasuwan, L. S. Chou, W. L. Clement, A. Couloux, B. Cousins, G. Genson, R. D. Harrison, P. E. Hanson, M. Hossaert-Mckey, R. Jabbour-Zahab E. Jousselin, C. Kerdelhué, F. Kjellberg, C. Lopez-Vaamonde, J. Peebles, Y. Q. Peng, R. A. Santinelo Pereira, T. Schramm, R. Ubaidillah, S. Van Noort, G. D. Weiblen, D. R. Yang, A. Yodpinyanee, R. Libeskind-Hadas, J. M. Cook, J. Y. Rasplus, and V. Savolainen. 2012. An extreme case of plant-insect codiversification: Figs and fig-pollinating wasps. Systematic Biology 61:1029-1047.

Darwin, C. 1859. On the Origin of Species. 1st ed. John Murray, London.

Davey, J. W., P. a. Hohenlohe, P. D. Etter, J. Q. Boone, J. M. Catchen, and M. L. Blaxter. 2011. Genome-wide genetic marker discovery and genotyping using next-generation sequencing. Nature Reviews Genetics 12:499-510.

De Vienne, D. M., G. Refrégier, M. López-Villavicencio, A. Tellier, M. E. Hood, and T. Giraud. 2013. Cospeciation vs hostshift speciation: Methods for testing, evidence from natural associations and relation to coevolution.

Drès, M., and J. Mallet. 2002. Host races in plant-feeding insects and their importance in sympatric speciation. Philosophical Transactions of the Royal Society B 357:471-492.

Ehrlich, P., and P. Raven. 1964. Butterflies and plants: A study in coevolution. Evolution 18:586-608.

Escudero, M. 2015. Phylogenetic congruence of parasitic smut fungi (Anthracoidea, anthracoideaceae) and their host plants (Carex, cyperaceae): Cospeciation or host-shift speciation?
American Journal of Botany 102:1108-1114.

Evans, L. M., G. J. Allan, N. Meneses, T. L. Max, and T. G. Whitham. 2013. Herbivore host-associated genetic differentiation depends on the scale of plant genetic variation examined. Evolutionary Ecology 27:65-81.

Farrell, B. D. 1998. "Inordinate fondness" explained: Why are there so many beetles? Science 281:555-559.

Farrell, B. D., C. Mitter, and D. J. Futuyma. 1992. Diversification at the Insect-Plant Interface. BioScience 42:34-42.

Fick, S. E., and R. J. Hijmans. 2017. WorldClim 2: new 1-km spatial resolution climate surfaces for global land areas. International Journal of Climatology 37:4302-4315.

Forbes, A. A., T. H. Powell, L. L. Stelinski, J. J. Smith, and J. L. Feder. 2009. Sequential sympatric speciation across trophic levels. Science 323:776-779.

Futuyma, D. J., and A. A. Agrawal. 2009. Macroevolution and the biological diversity of plants and herbivores. Proceedings of the National Academy of Sciences 106:18054-18061.

Futuyma, D. J., and S. C. Peterson. 1985. Genetic variation in the use of resources by insects. Annual Review of Entomology 30:217-238.

Garrido, E., G. Andraca-Gómez, and J. Fornoni. 2012. Local adaptation: simultaneously considering herbivores and their host plants. New Phytologist 193:445-453.

Gomulkiewicz, R., J. N. Thompson, R. D. Holt, S. L. Nuismer, M. E. Hochberg, B. Sciences, and P. O. Box. 2000. Hot spots, cold spots, and the geographic mosaic theory of coevolution. American Naturalist 156:156-174.

Grant, V. 1949. Pollination systems as isolating mechanisms in angiosperms. Evolution 3:82-97.

Hanks, L. M., and R. F. Denno. 1994. Local adaptation in the armored scale insect Pseudaulacaspis pentagona (Homoptera; Diaspididae). Ecology 75:2301-2310.

Hargreaves, A. L., R. M. Germain, M. Bontrager, J. Persi, and A. L. Angert. 2020. Local Adaptation to Biotic Interactions: A Meta-analysis across Latitudes. The American Naturalist 195:395-411.

Harrison, T. L., C. W. Wood, K. D. Heath, and J. R. Stinchcombe. 2017. Geographically structured genetic variation in the Medicago lupulina - Ensifer mutualism. Evolution 71:1787-1801.

Hembry, D. H., J. B. Yoder, and K. R. Goodman. 2014. Coevolution and the diversification of life. The American Naturalist 184:425-438.

Hijmans, R. J. 2020. raster: Geographic Data Analysis and Modeling. $R$ package version 3.3-13.

Hoeksema, J. D., and S. E. Forde. 2008. A Meta-Analysis of Factors Affecting Local Adaptation between Interacting Species. The American Naturalist 171:275-290.

Janz, N. 2011. Ehrlich and Raven revisited: Mechanisms underlying codiversification of plants and enemies. Annual Review of Ecology, Evolution, and Systematics 42:71-89.

Jerome, C. A., and B. A. Ford. 2002. Comparative population structure and genetic diversity of Arceuthobium americanum (Viscaceae) and its Pinus host species: insight into host-parasite evolution in parasitic angiosperms. Molecular Ecology 11:407-420.

Jost, L. 2008. G ST and its relatives do not measure differentiation. Molecular Ecology 17:4015-4026.

Kiester, A. R., R. Lande, and D. D. W. Schemske. 1984. Models of coevolution and speciation in plants and their pollinators. American Naturalist 124:220-243. 
Kopp, M., and S. Gavrilets. 2006. Multilocus genetics and the coevolution of quanitative traits. Evolution 60:1321-1336.

Kuhn, M., F. Chow, and H. Wickham. 2020. rsample: General Resampling Infrastructure. $\mathrm{R}$ package version 0.0.8.

Laine, A.-L. 2005. Spatial scale of local adaptation in a plantpathogen metapopulation. Journal of Evolutionary Biology 18:930-938.

Liu, M., J. Zhang, Y. Chen, S. G. Compton, and X.-Y. Y. Chen. 2013. Contrasting genetic responses to population fragmentation in a coevolving fig and fig wasp across a mainlandisland archipelago. Molecular Ecology 22:4384-4396.

Magalhaes, I. S., G. Gleiser, A. M. Labouche, and G. Bernasconi. 2011. Comparative population genetic structure in a plant-pollinator/seed predator system. Molecular Ecology 20:4618-4630.

Malé, P.-J. G., C. Leroy, P. Humblot, A. Dejean, A. Quilichini, and J. Orivel. 2016. Limited gene dispersal and spatial genetic structure as stabilizing factors in an ant-plant mutualism. Journal of Evolutionary Biology 29:2519-2529.

Maliet, O., N. Loeuille, and H. Morlon. 2020. An individualbased model for the eco-evolutionary emergence of bipartite interaction networks.

Matsubayashi, K. W., I. Ohshima, and P. Nosil. 2010. Ecological speciation in phytophagous insects. Entomologia Experimentalis et Applicata 134:1-27.

McKenna, D. D., S. Shin, D. Ahrens, M. Balke, C. BezaBeza, D. J. Clarke, A. Donath, H. E. Escalona, F. Friedrich, H. Letsch, S. Liu, D. Maddison, C. Mayer, B. Misof, P. J. Murin, O. Niehuis, R. S. Peters, L. Podsiadlowski, H. Pohl, E. D. Scully, E. V. Yan, X. Zhou, A. Ślipiński, and R. G. Beutel. 2019. The evolution and genomic basis of beetle diversity. Proceedings of the National Academy of Sciences page 201909655.

Meirmans, P. G. 2020. GENODIVE version 3.0: Easy-to-use software for the analysis of genetic data of diploids and polyploids. Molecular Ecology Resources 20:1126-1131.

Meirmans, P. G., P. H. Van Tienderen, and P. Note. 2004. GENOTYPE and GENODIVE: two programs for the analysis of genetic diversity of asexual organisms. Molecular Ecology Notes 4:792-794.

Mitter, C., B. Farrell, B. Wiegmann, T. A. Naturalist, and N. Jul. 1988. The phylogenetic study of adaptive zones: has phytophagy promoted insect diversification? American Naturalist 132:107-128

Nosil, P., B. J. Crespi, and C. P. Sandoval. 2003. Reproductive isolation driven by the combined effects of ecological adaptation and reinforcement. Proceedings of the Royal Society of London. Series B: Biological Sciences 270:1911-1918.

Nuismer, S. L. 2006. Parasite local adaptation in a geographic mosaic. Evolution 60:24.

Nuismer, S. L., R. Gomulkiewicz, and B. J. Ridenhour. 2010. When is correlation coevolution? American Naturalist 175:525-537.

Nuismer, S. L., B. J. Ridenhour, and B. P. Oswald. 2007. Antagonistic coevolution mediated by phenotypic differences between quantitative traits. Evolution 61:1823-1834.

Nychka, D., R. Furrer, J. Paige, and S. Sain. 2017. fields: Tools for spatial data $\mathrm{R}$ package version 10.3.

Peterson, M. A., and R. F. Denno. 1998. The influence of dispersal and diet breadth on patterns of genetic isolation by distance in phytophagous insects. The American Naturalist 152:428-446.
R Core Team. 2020. R: A Language and Environment for Statistical Computing. R Foundation for Statistical Computing, Vienna, Austria.

Rausher, M. D. 1983. Ecology of host-selection behavior in phytophagous insects. Pages 223-257 in Variable Plants and Herbivores in Natural and Managed Systems. Elsevier.

Ren, Z., B. Zhu, D. Wang, E. Ma, D. Su, and Y. Zhong. 2007. Comparative population structure of Chinese sumac aphid Schlechtendalia chinensis and its primary host-plant Rhus chinensis. Genetica 132:103-112.

Ridenhour, B. J., and S. L. Nuismer. 2007. Polygenic traits and parasite local adaptation. Evolution pages 368-376.

Rousset, F. 1997. Genetic differentiation and estimation of gene flow from F-statistics under isolation by distance. Genetics 61:183-200.

Runquist, R., A. Gorton, J. Yoder, N. Deacon, J. Grossman, S. Kothari, M. Lyons, S. Sheth, P. Tiffin, and D. Moeller. 2020. Context dependence of local adaptation to abiotic and biotic environments: A quantitative and qualitative synthesis. American Naturalist 195.

Schneider, A. C., A. E. Colwell, G. M. Schneeweiss, and B. G. Baldwin. 2016. Cryptic host-specific diversity among western hemisphere broomrapes (Orobanche s.l., Orobanchaceae). Annals of Botany 118:1101-1111.

Stireman, J. O., J. D. Nason, S. B. Heard, and J. M. Seehawer. 2006. Cascading host-associated genetic differentiation in parasitoids of phytophagous insects. Proceedings of the Royal Society B: Biological Sciences 273:523-530.

Thompson, J. N. 2005. The Geographic Mosaic of Coevolution. University of Chicago Press, Chicago.

. 2013. Relentless Evolution. University of Chicago Press, Chicago.

Toju, H., S. Ueno, F. Taniguchi, and T. Sota. 2011. Metapopulation structure of a seed-predator weevil and its host plant in arms race coevolution. Evolution 65:1707-1722.

Triponez, Y., N. Arrigo, A. Espíndola, and N. Alvarez. 2015. Decoupled post-glacial history in mutualistic plant-insect interactions: insights from the yellow loosestrife ( Lysimachia vulgaris) and its associated oil-collecting bees (Macropis europaea and M. fulvipes ). Journal of Biogeography 42:630640.

Wang, I. J., and G. S. Bradburd. 2014. Isolation by Environment. Molecular Ecology 23:5649-5662.

Wright, S. 1943. Isolation by distance. Genetics 28:114-138.

Yoder, J. B. 2016. Understanding the coevolutionary dynamics of mutualism with population genomics. American Journal of Botany 103:1742-1752.

Yoder, J. B., and S. L. Nuismer. 2010. When does coevolution promote diversification? American Naturalist 176:802-817.

Yoder, J. B., C. I. Smith, D. J. Rowley, R. Flatz, W. K. W. Godsoe, C. S. Drummond, and O. Pellmyr. 2013. Effects of gene flow on phenotype matching between two varieties of Joshua tree (Yucca brevifolia; Agavaceae) and their pollinators. Journal of Evolutionary Biology 26:1220-1233.

Yu, H., E. Tian, L. Zheng, X. Deng, Y. Cheng, L. Chen, W. Wu, W. Tanming, D. Zhang, S. G. Compton, and F. Kjellberg. 2019. Multiple parapatric pollinators have radiated across a continental fig tree displaying clinal genetic variation. Molecular Ecology 28:2391-2405. 